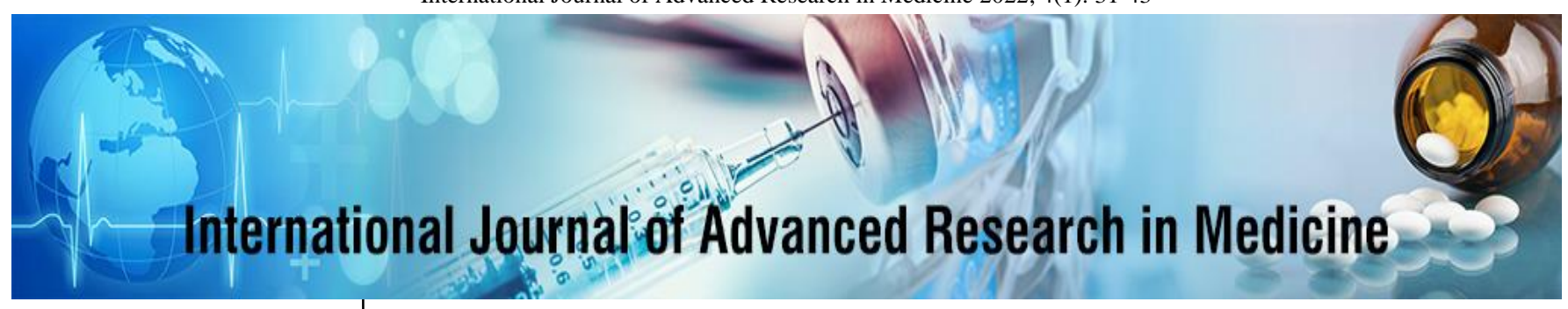

E-ISSN: 2706-9575

P-ISSN: 2706-9567

IJARM 2022; 4(1): 31-43

Received: 22-11-2021

Accepted: 24-12-2021

Dr. Dilshad Kauser

Assistant Professor,

Department of Anesthesia,

Bhasker Medical College.

Hyderabad, Telangana, India

\section{Anaesthesia for awake craniotomy in covid 19 recovered patient with cerebral mucormycosis}

\section{Dr. Dilshad Kauser}

DOI: $\underline{\text { https://doi.org/10.22271/27069567.2022.v4.i1a.320 }}$

\begin{abstract}
A 71-year-old male patient with cerebral Mucor mycosis was referred from a local COVID-19 treatment facility. Written informed consent was obtained from the patient for publication of the patients' details and images. The patient had an awake craniotomy and had a gross total tumour excision. There was no need to switch to general anaesthesia, The operation lasted four hours and thirty minutes. Throughout the awake craniotomy, the patient was haemodynamically stable, calm, cooperative, and comfortable. Amphotericin infusion administered at the end of the surgery, and Dexmedetomidine infusion administered after skin closure and the patient was completely awake and responsive when he was transferred out of the operating room and the patient's neurological condition improved post-surgery. On post-operative day 5, a repeat COVID-19 test came out negative, and the patient was discharged. A thirty-day post-operative assessment confirmed the patient's increasing motor gain. Although maintaining analgesia and hemodynamic stability with the patient awake was challenging, a target-controlled propofol infusion gave the required level of awareness, fentanyl titrated analgesia and sedation without drug accumulation, and bupivacaine blockade provided appropriate analgesia. We came to the conclusion that our patient's anaesthetic method was effective and It is possible to do an awake craniotomy in a COVID-19 positive patient with the requisite PPEs.
\end{abstract}

Keywords: awake craniotomy, mucor mycosis, lesion, Covid-19

\section{Introduction}

Awake craniotomy is used to detect and remove epileptic foci, as well as tumours near the eloquent regions of the brain such Broca's speech area, Wernicke's speech area, and the motor strip region. It enables intraoperative neurological assessment, allowing for optimum tumour excision and the reduction of post-operative neurological impairment. However, both the neurosurgeon and the anaesthesiologist have difficulties with this approach ${ }^{[1]}$.

The objective of anaesthesia is to ensure awake, comfortable, and cooperative patient for accurate neurological testing, in addition to maintaining acceptable sedation, analgesic, respiratory, and haemodynamic stability. Over the last two decades, there has been an increasing tendency toward preferring awake craniotomy as a technique, and we also have jumped on the bandwagon. There is always a worry regarding the patient's willingness to cooperate, as well as their safety, throughout this treatment ${ }^{[2]}$.

Such operations require careful patient selection, counselling, appropriate measures to keep the patient comfortable throughout surgery, and effective communication with the patient. Despite all of the foregoing precautions, a variety of perioperative adverse effects have been documented, necessitating intervention. Due to the patient's non-cooperation or agitation, the procedure may need to be switched to general anaesthesia (GA) ${ }^{[3,4]}$.

The COVID-19 infection is caused by the SARS-COV-2 virus, which is a novel coronavirus ${ }^{[5]}$. It is an infectious illness with a virus whose biology is unknown ${ }^{[6,7]}$. Though the virus began in late 2019 in China's Wuhan province, it has since spread tremendously across the globe, resulting in one of the most terrifying pandemics in human history. The novel coronavirus poses a twin threat: 1) direct mortalities and morbidities attributed with it, and 2) the influence of its pandemic on the health-care delivery system, resulting in indirect mortalities and morbidities on patients who were unable to receive appropriate and timely medical assistance ${ }^{[8,9]}$.

The coronavirus disease pandemic of 2019 (COVID-19) is continuing to spread. The disease's second wave has caused panic in several nations, including India and certain
Dr. Dilshad Kauser

Assistant Professor,

Department of Anesthesia,

Bhasker Medical College.

Hyderabad, Telangana, India 
of the world where the third wave is raging. Supportive care equipment such as oxygen cylinders, ventilators, and extensive usage of steroids are essential in the management of COVID-19 since there are no effective treatment options orremedies for this deadly infection. COVID-19 individuals are contracting secondary illnesses such as Mucor mycosis, often known as black fungus disease, in the midst of the epidemic. Mucor mycosis is a serious yet rare opportunistic fungal infection that spreads rapidly necessitating timely diagnosis and treatment to minimize high mortality and morbidity rates. Mucor mycosis is caused by inhaling filamentous (hyphal form) fungus, which is particularly problematic in immunocompromised people. Moulds may be found all over the place. After inhaling fungal spores from the air, it most frequently affects the sinuses or lungs. It can also happen as a result of a cut, a burn, or another form of skin damage ${ }^{[10]}$.

The COVID-19 epidemic is also having an impact on the neurosurgical field. Awake craniotomy allows for optimum tumour excision in the eloquent brain area with the best possible outcome while also reducing the patient's exposure to general anaesthetics and their side effects.

Awake craniotomy in a COVID-19 positive patient poses a considerable hazard to theatre staff, but intubation for general anaesthesia in a COVID-19 positive patient poses a similar risk due to the virus's high concentration in the respiratory tract ${ }^{[11]}$. The goal of this report is to highlight the difficulties and outcomes of our first awake craniotomy in a COVID-19 recovered patient.

\section{Case Presentation}

A 71-year-old male weighing $65 \mathrm{kgs}$ and measuring at 165 cms was brought to the casualty ward in a drowsy state with altered sensorium. The patient had Covid-19 two months ago, and after a month of recuperation, he was diagnosed with Mucor mycosis of the nose and sinuses and had undergone Functional Endoscopic Surgery (FES) and Right Maxillectomy.

The patient had uncontrolled diabetes mellitus and was also asthmatic.

Airway examination: Facial structure was found to be distorted following a complete (right) maxillectomy. Mouth opening was found to be $2 \mathrm{Fb}$ (Finger breadths), Edentulous, Nostril distorted, asymmetrical, Mallam Pati grade IV (Soft palate not visible at all).

The tongue is coated in white secretions, and the nasal passages are clogged with secretions. With a stiff neck and neck extension was limited.

Pre-procedure: Upon physical examination, the patient was conscious, responsive and obeying commands, had a speech defect, and generalized pitting oedema and pallor.

Since midnight, the patient has been kept nil by mouth. Tab. Ranitidine 150mg was administered through Ryle's tube the night before surgery, and FBS was tested prior to surgery.

\section{Investigations If relevant}

- 2D Echo: Normal limits

- Hb, Renal and liver functions tests

- CT-Scan

After necessary lab investigations reports at disposal the patient was taken upon for the procedure.

\section{Differential Diagnosis}

Cerebral Mucor Mycosis

\section{Treatment}

A difficult airway cart and a crash cart were kept on standby in the operation room

ASA monitors were fixed, an $18 \mathrm{G}$ intravenous addition line was inserted, and a loading dose of $0.8 \mathrm{ugl}$ of dexmedetomidine was administered during a 15-minute period (dose and duration modified).

Using another IV Cannula Inj. Paracetamol 1gm, Inj. Mannitol, IV Levipil were administered as slow fusion. Oxygen was supplemented through a face mask and an End-tidal $\mathrm{CO} 2(\mathrm{EtCO} 2)$ probe was inserted within the mask as an addition to the respiratory monitor and respiratory rate probe.

The nerves on the left side of the scalp were numbed with a scalp block, with $20 \mathrm{ml}$ of $0.25 \%$ Bupivacaine $+10 \mathrm{ml} 2 \%$ Xylocaine $+2 \mathrm{ml}$ Dexamethasone $+10 \mathrm{ml}$ distil water.

An IV fluid of $50 \mathrm{~mL} / \mathrm{hr}$ isotonic saline was administered, and the general random blood sugar (GRBS) level was observed to be $200 \mathrm{mg} / \mathrm{dl}$.

After 15 minutes of Dexamethasone infusion, the rate was reduced to $0.4 \mathrm{mg}$, and $20 \mathrm{mg}$ Fentanyl+10mg Propofol was administered to incision.

The operation lasted four hours and thirty minutes.

Throughout the awake craniotomy, the patient was haemodynamically stable, calm, cooperative, and comfortable.

Amphotericin infusion administered at the end of the surgery, and Dexmedetomidine infusion administered after skin closure and the patient was completely awake and responsive when he was transferred out of the operating room.

\section{Outcome And Follow-Up}

On post-operative day 5, the nasal swab for the RT-PCR test was repeated. The patient was discharged when the repeat RT-PCR test came out negative. A thirty-day post-operative assessment revealed significant motor improvement. He was standing with minimal support.

\section{Discussion}

Modern anaesthesia necessitates the anaesthesiologist's grasp of medical knowledge in order to make vital decisions in a short amount of time. The operating room's equipment and settings help in thorough management of anaesthetic, whether it's inhaled, mixed, or entirely venous. Although awake craniotomy is well-known, it has only lately gained popularity for tumour surgery.

The COVID-19 epidemic has been ongoing for more than a year. Both developed and emerging countries, including our own, are bearing the effects. Patients both with COVID-19 positive and negative results encounter difficulties as a consequence of the pandemic. There are debates over perioperative care, with many advocating for routine COVID-19 screening for all surgical patients to avoid unnecessarily exposing health-care personnel to the virus. Some have recommended that all surgery patients wear complete PPEs, however this is not possible owing to a lack of supplies that has affected nearly every country on the planet. The initial goal of postponing all elective neurosurgery cases is no longer feasible, and we must modify our approach to improve patient outcomes. 
In our study, dexmedetomidine was used with fentanyl. Ard et al. reported no serious complications in 17 patients who had undergone awake craniotomy with dexmedetomidine infusion ${ }^{[12]}$. There were no complications in our case.

Blanshard et al. reported the anaesthetic treatment and sequelae of neurolept anaesthesia in 241 awake patients with intracranial tumours. Because of widespread convulsion with loss of airway patency, one patient $(0.4 \%)$ needed conversion to $\mathrm{GA}^{[13]}$.

Our patient did not have postoperative nausea and vomiting. It's feasible because the patient was given antiemetic medicine prior to the procedure. Furthermore, the administration of steroids as anti-oedema measures and propofol for sedation may have contributed to the prevention of such occurrences. In Few studies, the incidence of nausea and vomiting has been found to be as high as $70 \%$. This high incidence might be explained by the omission of propofol in the anaesthetic regimen ${ }^{[14]}$.

Using awake craniotomy in the COVID-19 era, we were able to accomplish optimum tumor excision with no additional morbidity. The intraoperative monitoring was a significant area of concern since the patient's usage of a face mask restricts clinical observation. The COVID-19 pandemic might persist longer than expected, therefore we should adapt and modify our practices to ensure that patients receive the best possible treatment in the face of this lethal virus.

\section{Learning Points/Take Home Messages}

- Anaesthesia for awake craniotomy (AC) is an approach that has evolved over time by using current cortical mapping techniques, drug research, and continuous infusion methods. It enables for ongoing evaluation of the patient's neurological functioning, resulting in a greater degree of brain tumour excision and functional neurological preservation.

- The use of a scalp block provides comfort to the patient while also minimizing the need of anaesthetic drugs during the procedure and ensuring tight pain management in the postoperative period.

- This procedure allows for shorter hospital stays and early return to normal life.

- This anaesthetic method is being refined in order to enhance the prognosis of neuro-oncological patients and to allow other practitioners to replicate it in their own settings.

- It is possible to do an awake craniotomy in a COVID19 recovered patient with the requisite PPEs.

- The main anaesthetic concern in the patient was difficult airway due to maxillectomy(right side), post covid lung disease, uncontrolled diabetes mellitus, Asthma, Altered sensorium, high risk craniectomy surgery. We successfully manage awake craniectomy with unilateral scalp block and conscious sedation with dexmedetomidine infusion through out the surgery. The patient was calm, comfortable and co-operative with utmost satisfaction of the operating surgeon for prolong duration of four hours. Post-operative course was well managed with anti-fungal drugs and anti-cerebral oedema drugs and discharged after 10 days of hospital stay post-operatively.

- So the scalp block with conscious sedation with cocktail drugs predominantly dexmedetomidine infusion is effective alternative form of anaesthesia technique for life saving craniectomy.

\section{Figure/Video Captions}

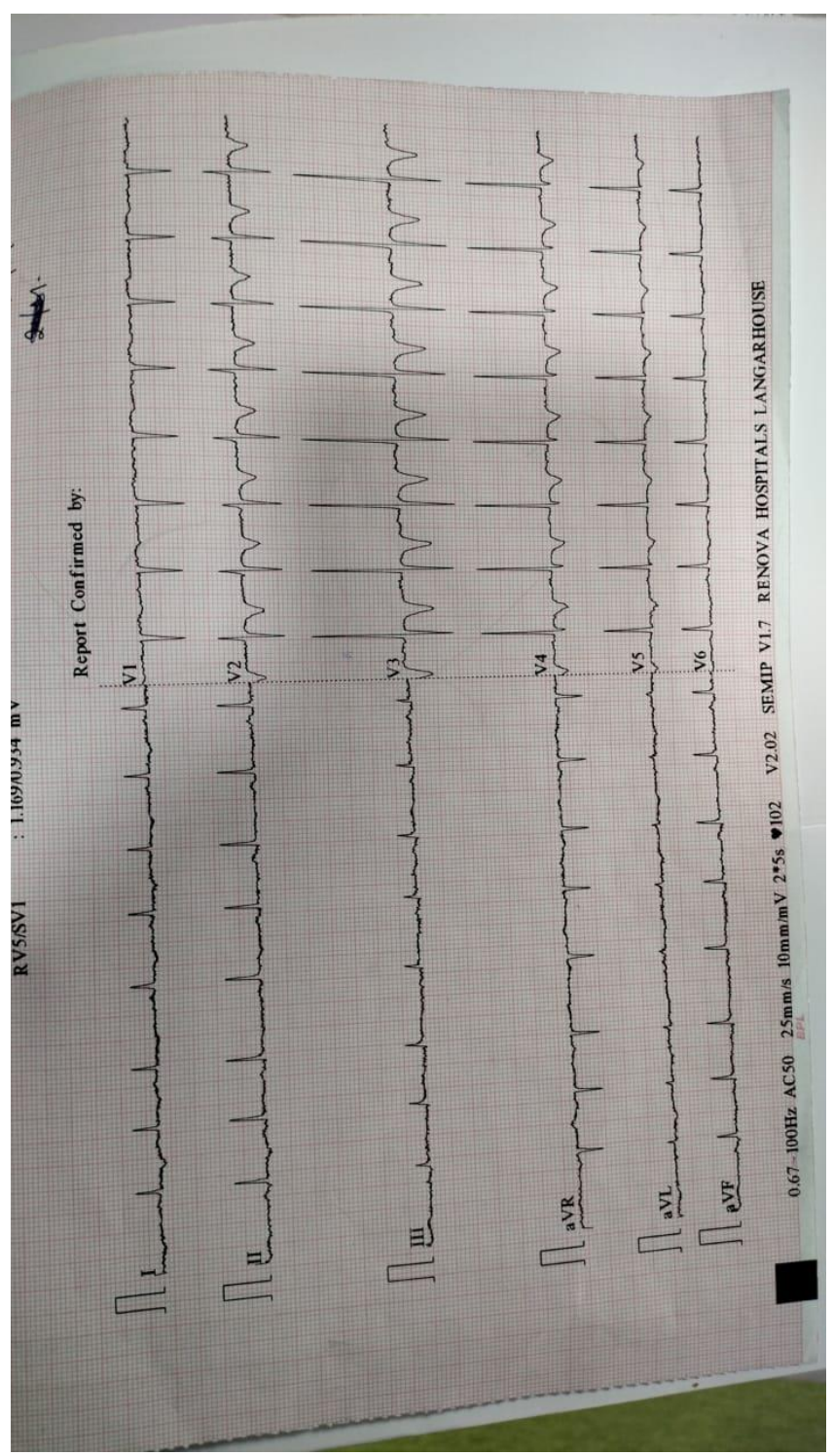

Fig 1: ECG- Normal sinus rhythm with LVH strain pattern 


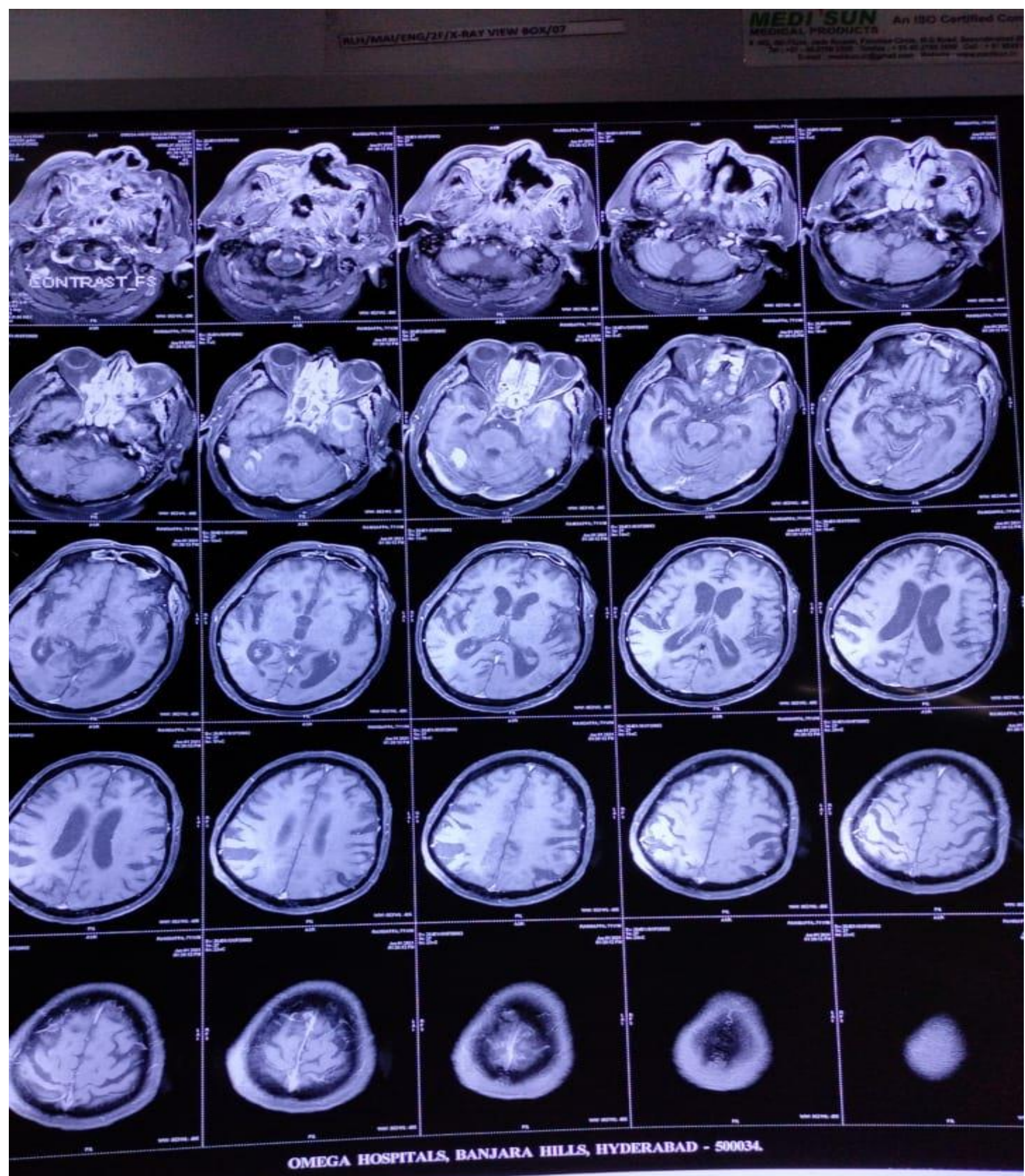

Fig 2: CT-scan - Rhinocerebral mucormycosis post maxillectomy and temporal lobe lesion 

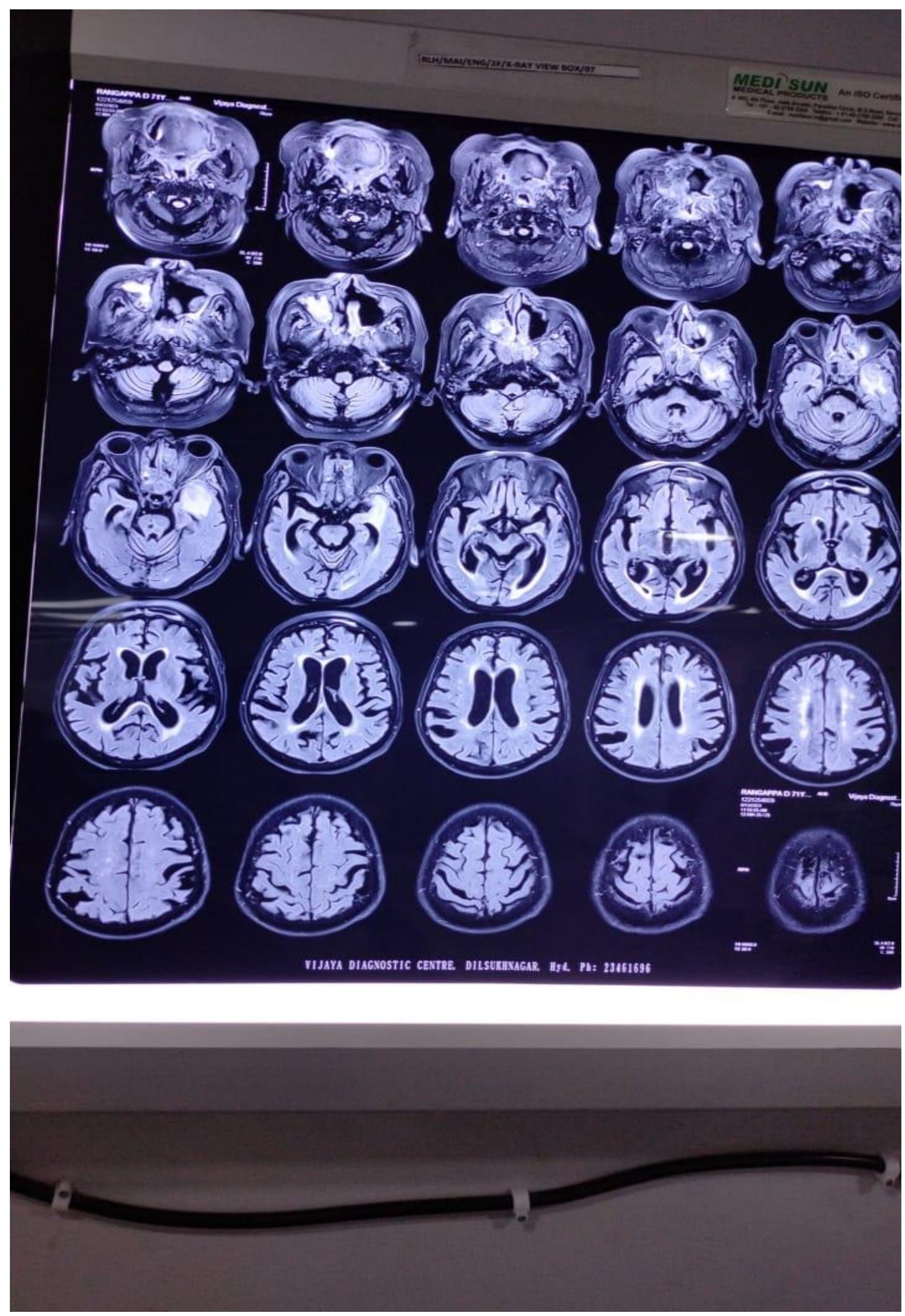

Fig 3: CT-scan - Rhinocerebral mucormycosis post maxillectomy and temporal lobe lesion 


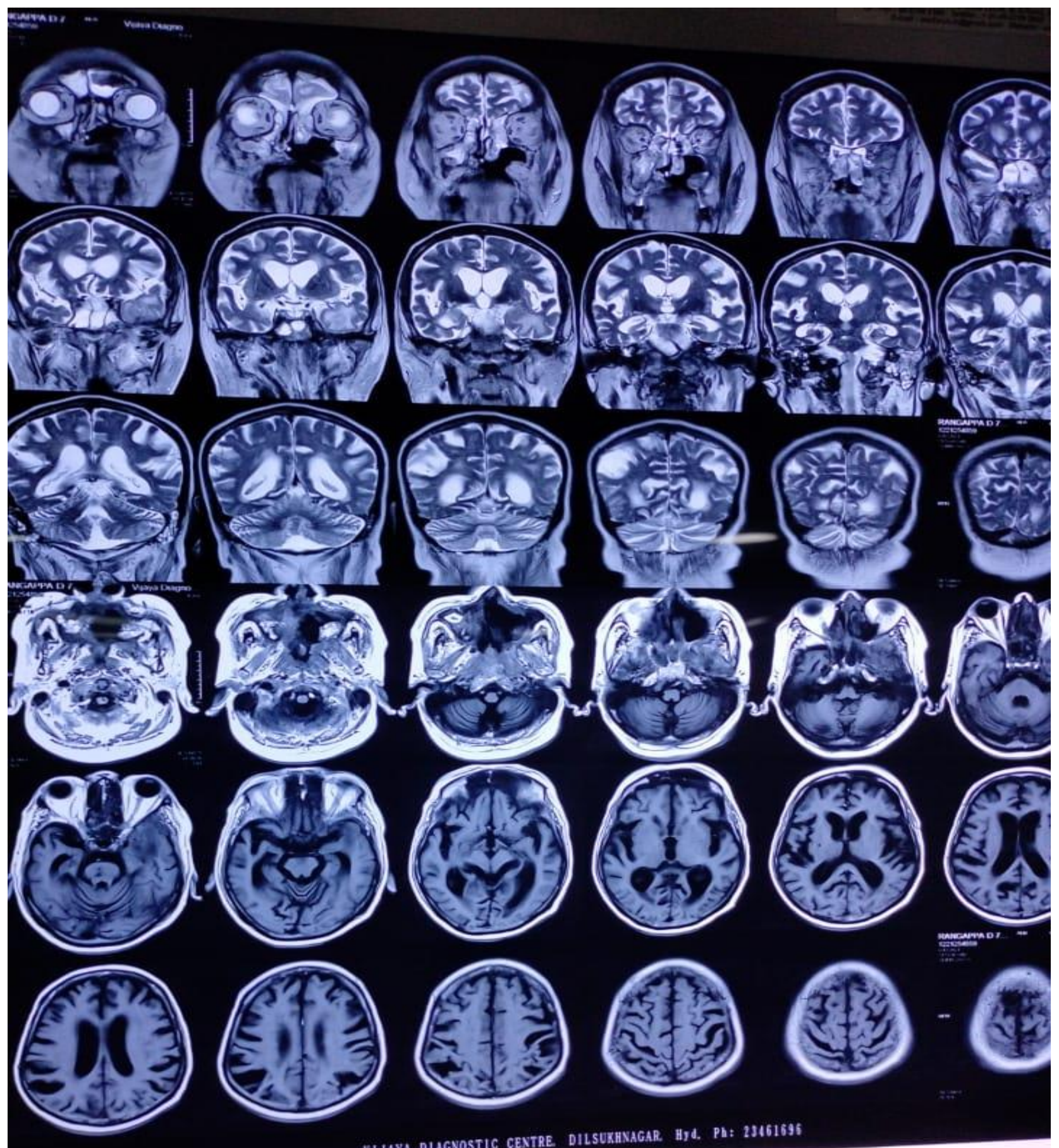

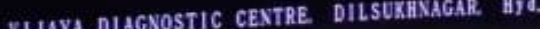



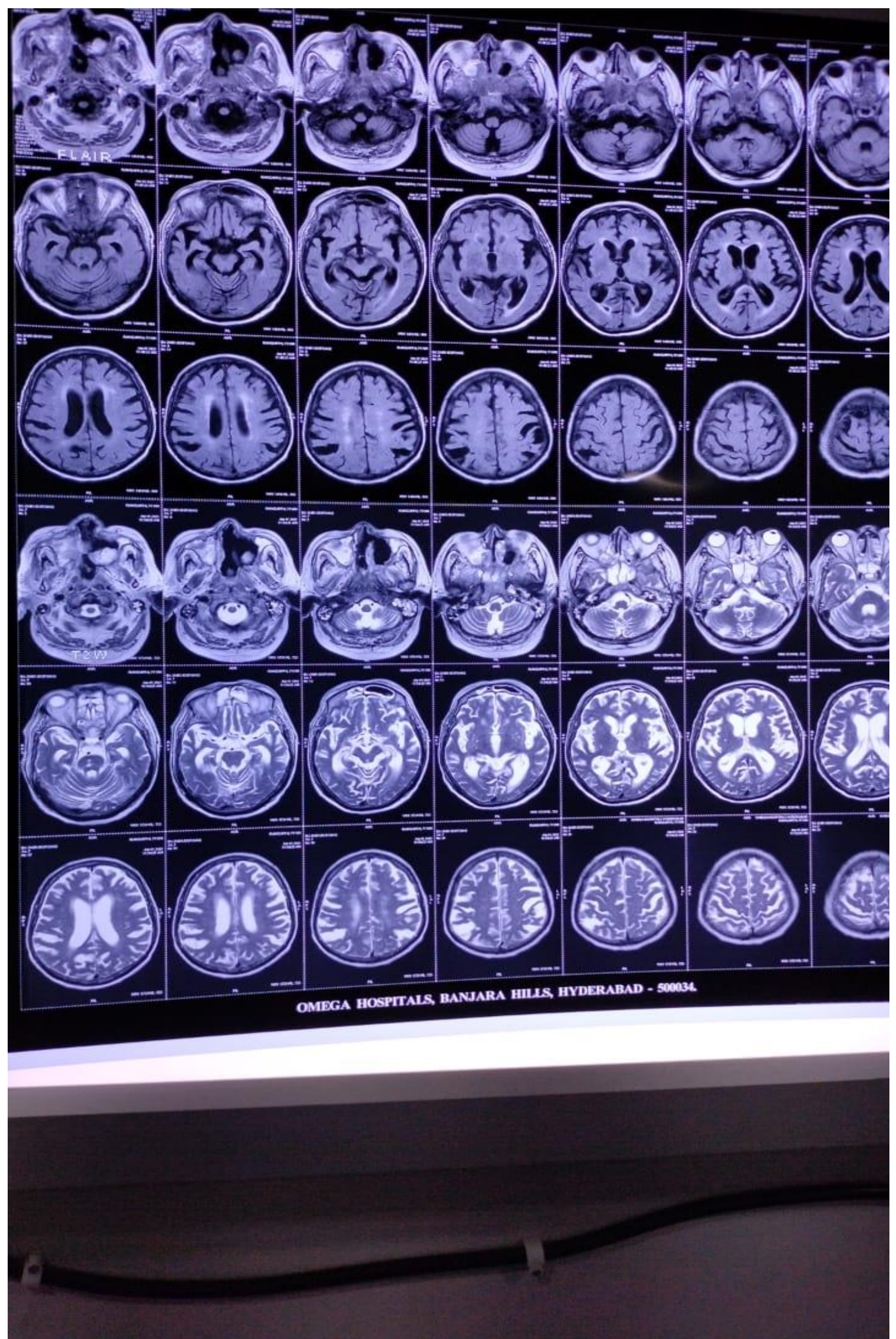

Fig 5: CT-scan - Rhinocerebral mucormycosis post maxillectomy and temporal lobe lesion 


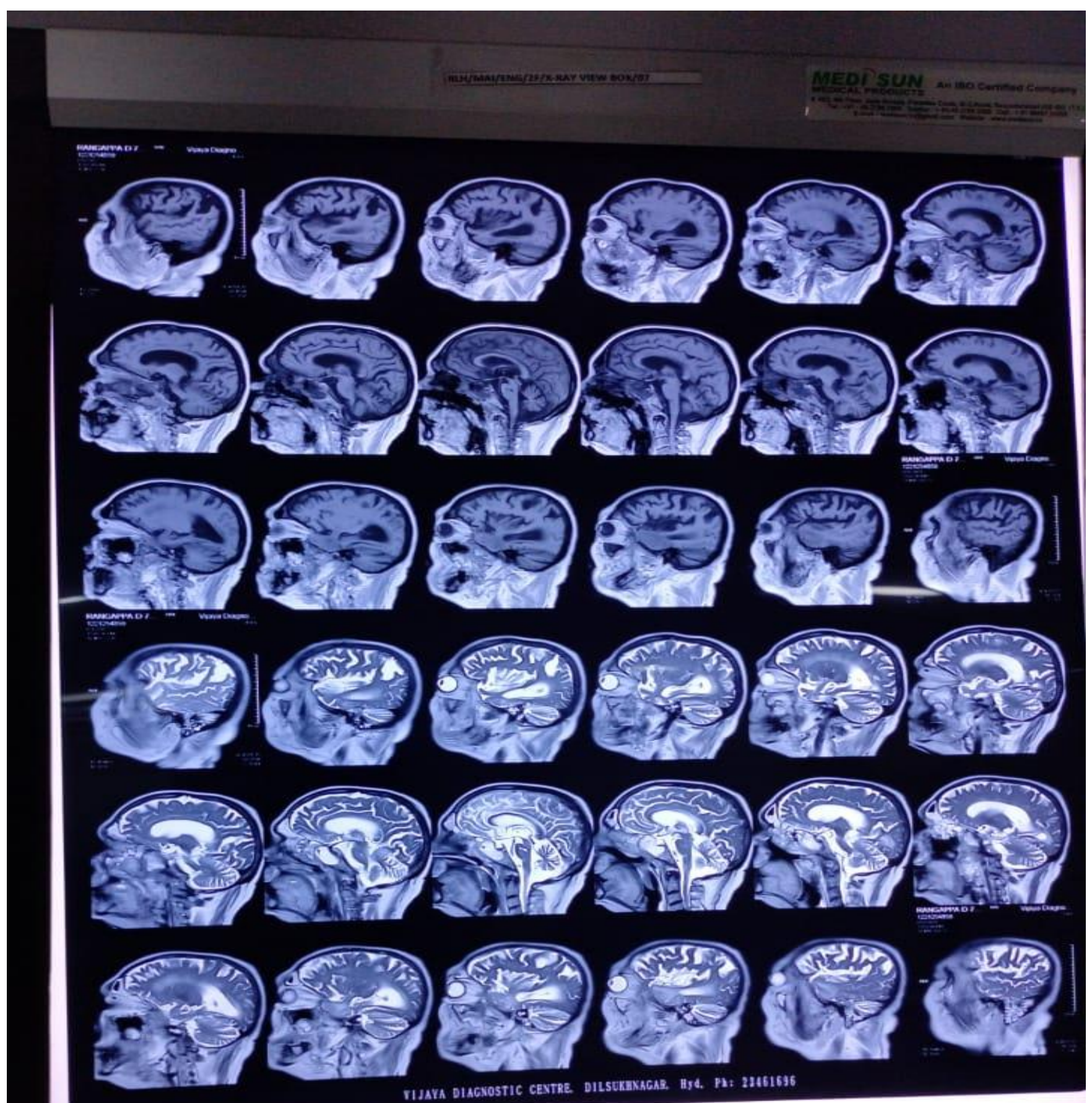

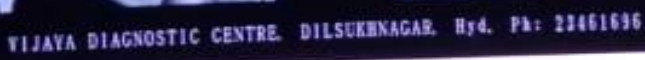




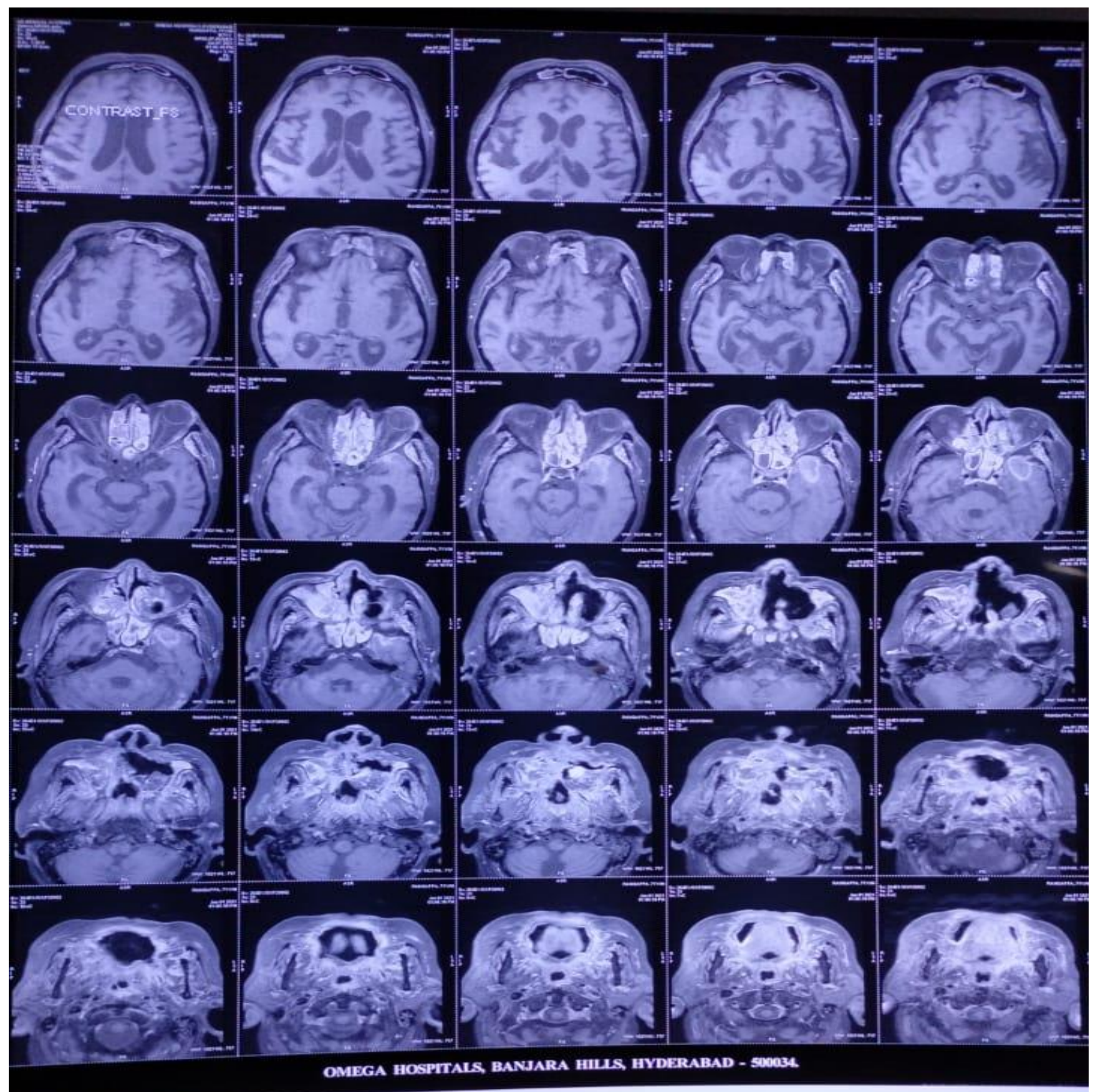

Fig 7: CT-scan - Rhinocerebral mucormycosis post maxillectomy and temporal lobe lesion 


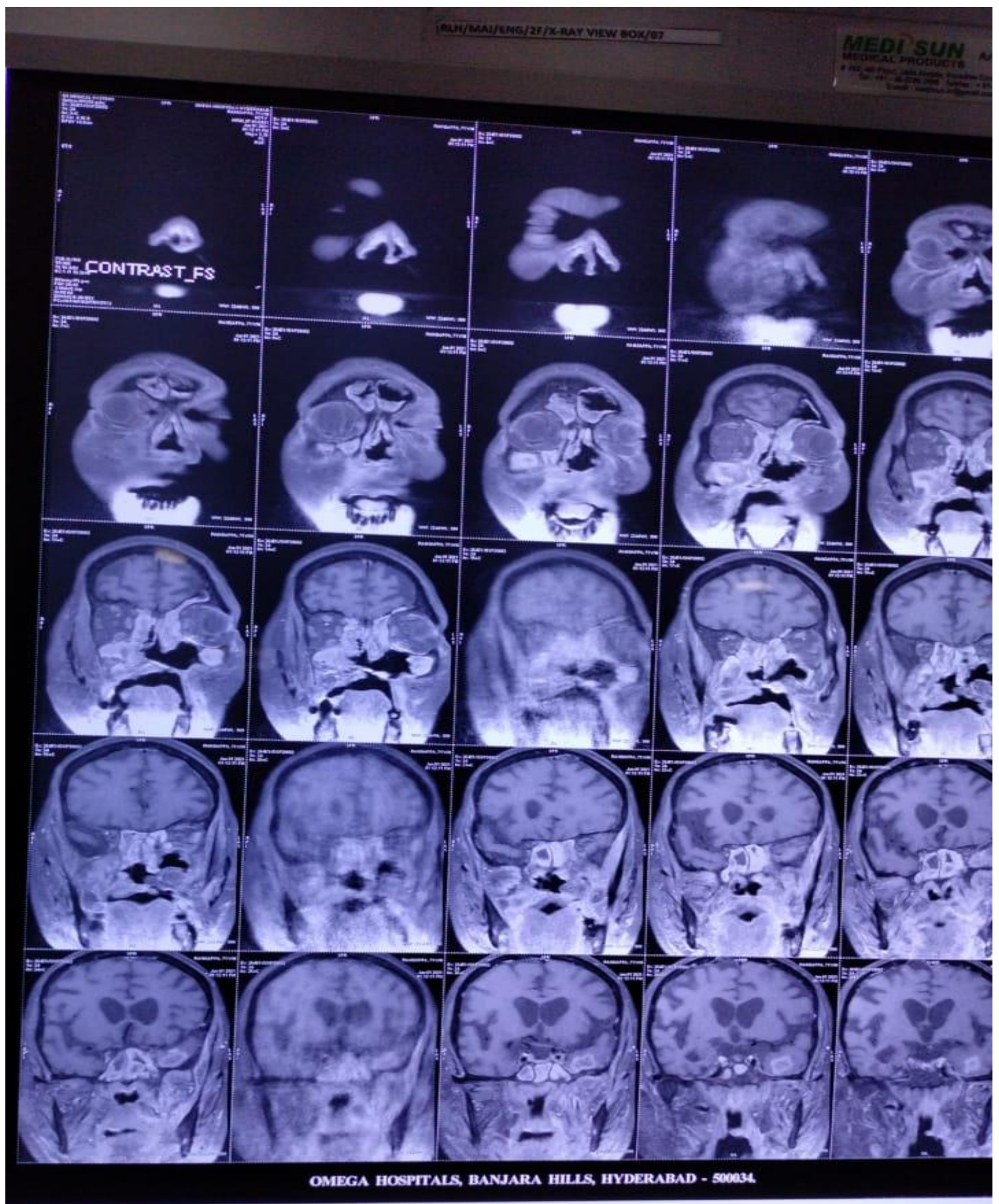

OMEGA HOSPTALS, BANJARA HILL, HYDERABAD - 500034

Fig 8: CT-scan - Rhinocerebral mucormycosis post maxillectomy and temporal lobe lesion 


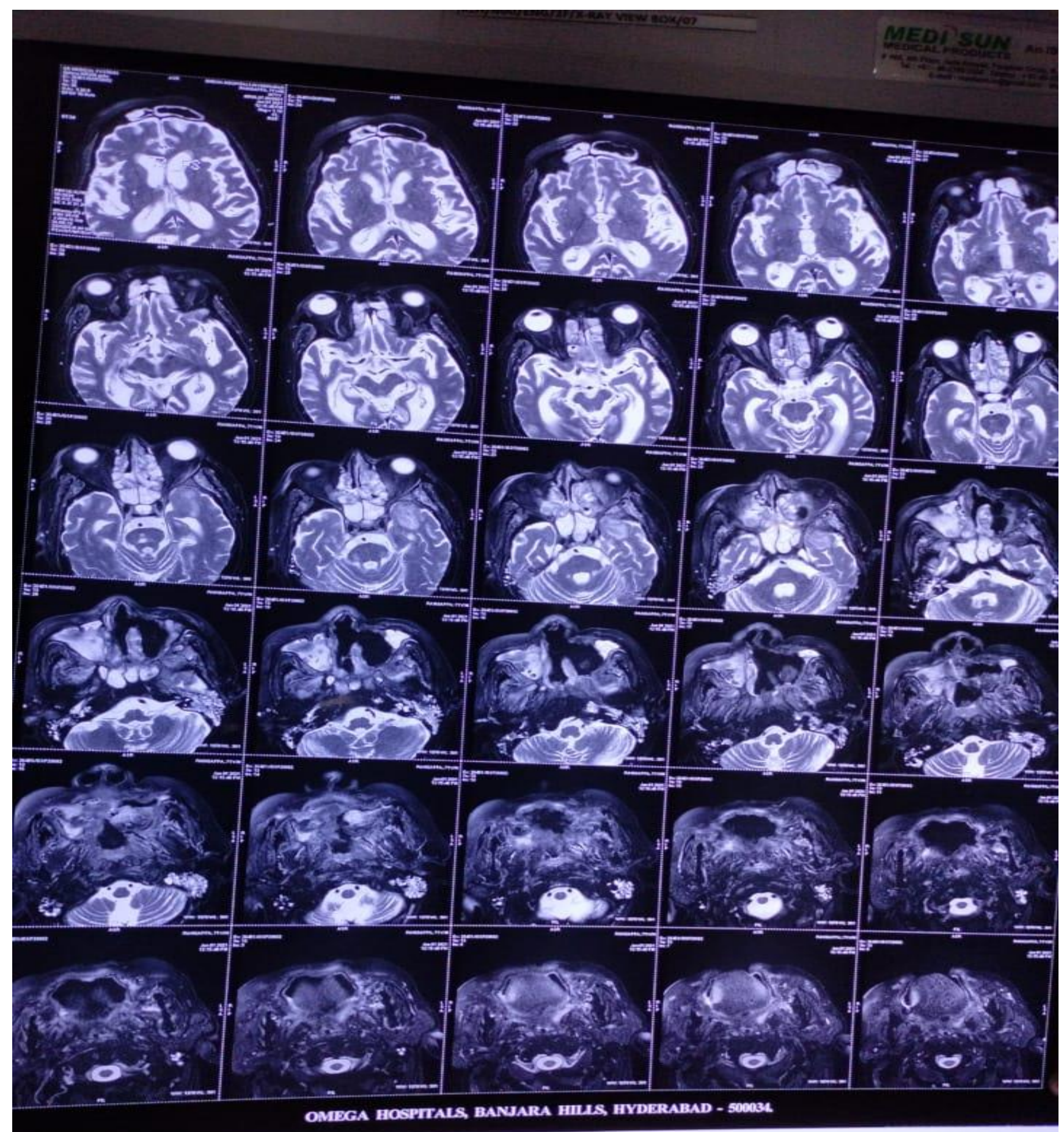

\footnotetext{
OMISGA hOSPTTALS, BANUARA HILL, HYDERABAD - 500034
}

Fig 9: CT-scan - Rhinocerebral mucormycosis post maxillectomy and temporal lobe lesion 


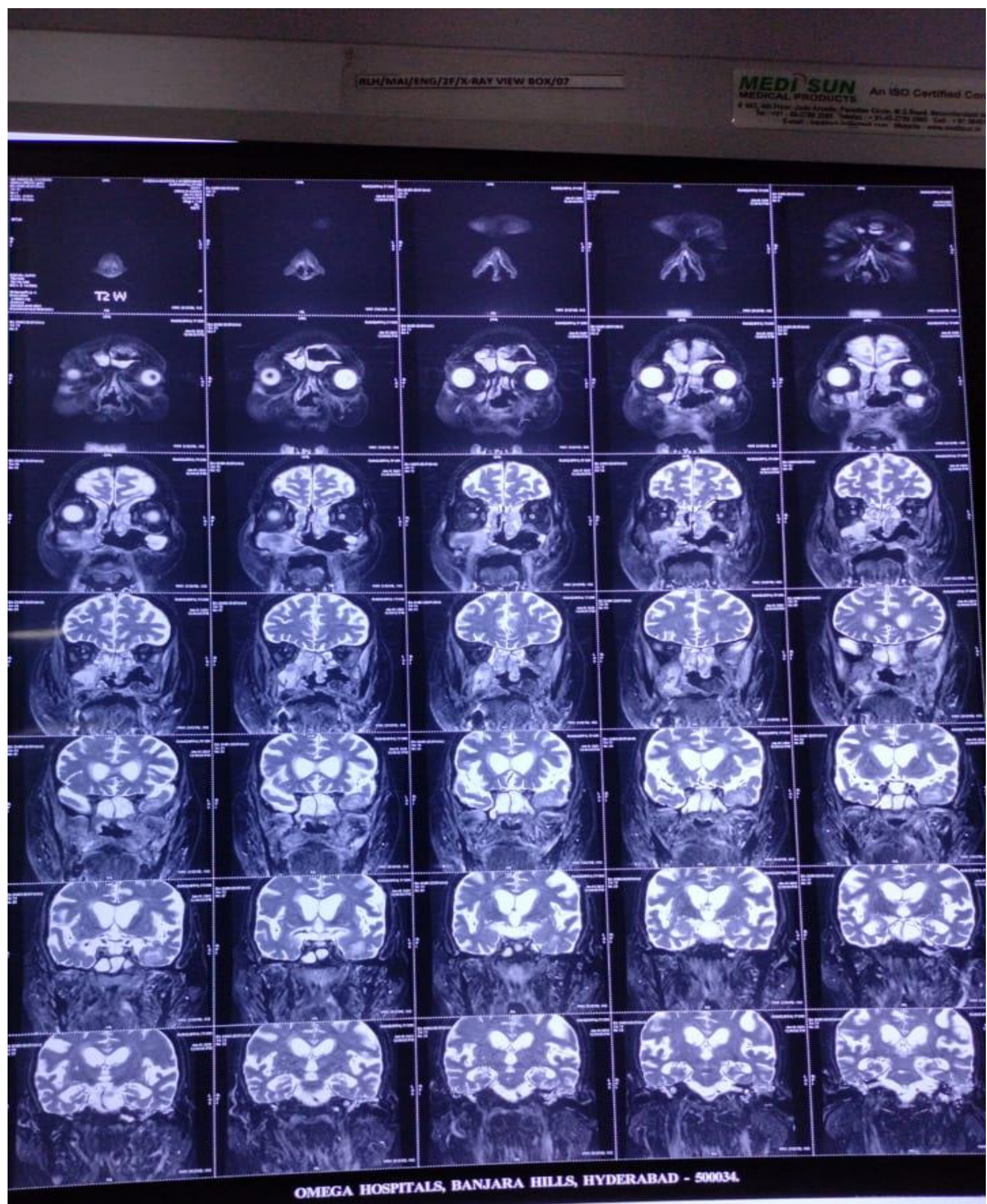

Fig 10: CT-scan - Rhinocerebral mucormycosis post maxillectomy and temporal lobe lesion 


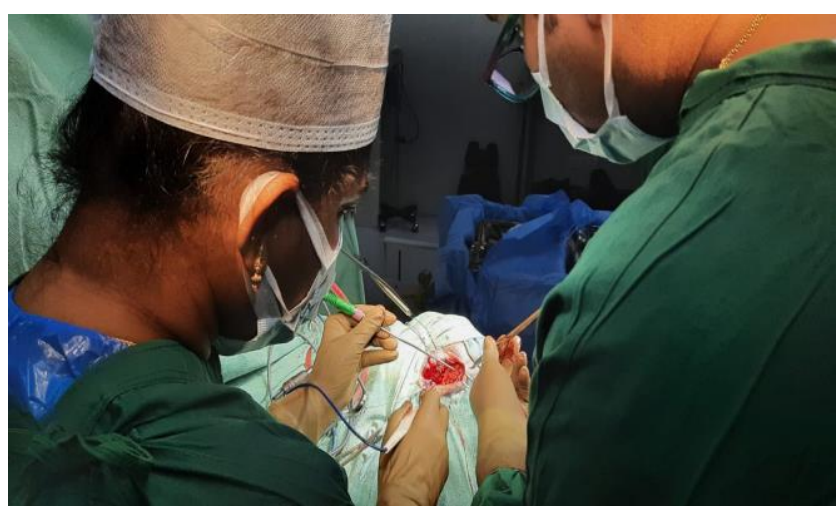

Fig 11: Rhinocerebral mucormycosis post maxillectomy right side and temporal lobe lesion

\section{References}

1. Sahjpaul RL. Awake craniotomy: Controversies, indications and techniques in the surgical treatment of temporal lobe epilepsy. Can J Neurol Sci. 2000;27(1):S55-63.

2. Danks RA, Rogers M, Aglio LS, Gugino LD, Black PM. Patient tolerance of craniotomy performed with the patient under local anesthesia and monitored conscious sedation. Neurosurgery. 1998;42:28-34

3. Whittle IR, Midgley S, Georges H, Pringle AM, Taylor R. Patient perceptions of "awake" brain tumour surgery. Acta Neurochir (Wien). 2005;147:275-7.

4. Piccioni F, Fanzio M. Management of anesthesia in awake craniotomy. Minerva Anestesiol. 2008;74:393408.

5. Khan S, Siddique R, Shereen A, Ali A, Liu J, Bai Q. Emergence of a novel coronavirus, severe acute respiratory syndrome coronavirus 2: biology and therapeutic options. J Clin. Microbiol. 2020;58(5):111 .

6. Anastasopoulou S, Mouzaki A. The biology of SARSCoV-2 and the ensuing COVID-19. Achaiki Iatriki. 2020;39(1):29-35.

7. Adedokun KA, Olarinmoye AO, Mustapha JO, Kamorudeen RT. A close look at the biology of SARS$\mathrm{CoV}-2$, and the potential influence of weather conditions and seasons on COVID-19 case spread. Infect. Dis. Poverty. 2020;9(77):1-5.

8. Tsermoulas G, Zisakis A, Flint G, Belli A. Challenges to neurosurgery during the Coronavirus Disease 2019 (COVID-19)

Neurosurgery 2020;139:519-525.

doi: 10.1016/j.wneu.2020.05.108.

9. Healy DW, Cloyd BH, Brenner MJ, Kupfer RA, Anam KS, Schechtman SA. The COVID-19 pandemic: implications for the head and neck anesthesiologist. J. Head Neck Anesth. 2020;4(e26):1-8.

10. Adil A. Anadolu Agency; Over 28,200 'black Fungus' Cases Recorded in India 2021.

https://www.aa.com.tr/en/asia-pacific/over-28-200black-fungus-cases-recorded-in-india/2266396 2021.

11. Snacc CC, Flexman AM, Abcejo AS, Avitsian R, De $S V$, Highton D. Neuroanesthesia practice during the COVID-19 pandemic: recommendations from society for euroscience. J Neurosurg. Anesth 2020;32(3):202209.
12. Ard JL Jr, Bekker AY, Doyle WK. Dexmedetomidine in awake craniotomy: a technical note. Surg Neurol. 2005;63(2):114-6 discussion 116-7.

13. Blanshard HJ, Chung F, Manninen PH, Taylor MD, Bernstein M. Awake craniotomy for removal of intracranial tumor: considerations for early discharge. Anesth Analg. 2001;92(1):89-94.

14. Gignac E, Manninen PH, Gelb AW, Comparison of fentanyl, sufentanil and alfentanil during awake craniotomy for epilepsy. Can J Anaesth. 1993;40(5 Pt $1): 421-4$ 